\title{
Thermal Temperature Measurements of Plasma Torch by Alexandrite Effect Spectropyrometer
}

\author{
Peir-Jyh Wang, ${ }^{1}$ Chin-Ching Tzeng, ${ }^{1}$ and Yan Liu ${ }^{2}$ \\ ${ }^{1}$ Institute of Nuclear Energy Research, Atomic Energy Council, 1000 Wenhua Road, Taoyuan 32546, Taiwan \\ ${ }^{2}$ Liu Research Laboratories, 2202 Seaman Avenue, South El Monte, CA 91733, USA \\ Correspondence should be addressed to Yan Liu, yanliu@liulabs.com
}

Received 18 February 2010; Revised 15 June 2010; Accepted 5 July 2010

Academic Editor: Jagdish P. Singh

Copyright ( 2010 Peir-Jyh Wang et al. This is an open access article distributed under the Creative Commons Attribution License, which permits unrestricted use, distribution, and reproduction in any medium, provided the original work is properly cited.

\begin{abstract}
An alexandrite effect spectropyrometer is used to directly measure the thermal temperature of an argon gas plasma jet from a $100 \mathrm{~kW}$ DC plasma torch, and the directly measured thermal temperature of the plasma is $11178 \pm 382 \mathrm{~K}$. By using the spectral correction function to delete the spectral lines and to correct its underlying spectrum of the relative spectral power distribution of the plasma jet, the remaining continuum spectral power distribution represents the thermal spectral emission of the plasma jet. The calculated thermal temperature of the corrected relative continuum spectral power distribution by the spectropyrometer is $10106 \mathrm{~K} \pm 345 \mathrm{~K}$, which is the true thermal temperature of the plasma jet. The blackbody level (BL) of the thermal plasma jet is defined as the ratio of the true thermal temperature to the directly measured temperature, and the blackbody level is a measure of how well the thermal plasma jet approaches a blackbody. The accuracy of directly measured thermal temperature depends on the blackbody level, the higher the blackbody level, and the higher the thermal temperature measurement accuracy.
\end{abstract}

\section{Introduction}

Plasma jets generated in DC arc torches are used for spraying, cutting, synthesis, carbon material vaporization, and decomposition of persistent chemical substances. The temperature fluctuation of a plasma torch significantly reduces its reliability for industrial applications. The characteristics of emission spectra from the flame jet of a plasma torch directly indicate the plasma properties and operation conditions. It's a well-established technique [1-3] to measure the kinetic temperature using optical emission spectroscopy (OES) methods; however, none of the methods were accurate under temporal and spatial changing conditions.

Local Thermodynamic Equilibrium (LTE) is assumed for calculating the temperature from the populations of excited levels. The calculated temperature is based on the absolute and relative intensities of various atomic lines. The temperature is often calculated from the ratios of gas atomic to ionic line emission coefficients by the Saha equation using the measured electron number density or is calculated by the ratio of spectral lines by the Ornstein method. It assumes that collisions are predominant and the temperature and concentration gradients are low, which are not true in jet fringes or close to electrodes or substrates. The diffusion of particles and electrons plays an important role in the regions of the plasma. The temperatures calculated from the spectral lines are generally overestimated [4].

Planck's radiation law gives the spectral power distribution of a thermal radiator (blackbody), and the wavelength with the maximum spectral density obeys Wein's displacement law [5]. The Planck distribution forms a locus in the CIE color space, and the color of a thermal radiator changes from red, orange, yellow, and white to blue as temperature increases [6]. Temperature is commonly measured by radiation thermometers, such as total radiation thermometer, spectral-band radiation thermometer, wideband pyrometer, two-color pyrometer, and disappearing filament optical pyrometer.

The alexandrite effect refers to the color change phenomenon in alexandrite crystals between different light sources [7]. For example, an alexandrite gemstone can appear bluish green under daylight $(6500 \mathrm{~K})$ and purplish red under incandescent light $(2856 \mathrm{~K})$. The two colors in this example are opponent colors, with a hue-angle difference 
of about 180 degrees in the CIELAB color space. This is the largest possible color change for human color vision perception. Liu [8] also established the relationship between temperature of a thermal radiator (blackbody) and hue-angle in the CIELAB color space, introduced a novel alexandrite effect method, and developed an alexandrite effect spectropyrometer for the thermal temperature measurement.

In this paper, we use the alexandrite effect spectropyrometer to directly measure the thermal temperature of a plasma jet, and the results are compared with the kinetic temperature calculated by the optical emission spectroscopy (OES) method. We also use the spectral correction function of the spectropyrometer to delete all spectral lines and correct the underlying spectrum, and we calculate the true thermal temperature using the remaining relative continuum spectral power distribution that represents the thermal spectral emission of the plasma jet. In addition, we define the blackbody level (BL) of the thermal plasma jet to estimate how well the thermal plasma jet approaches a blackbody.

\section{Alexandrite Effect Method}

The "alexandrite effect" is used in gemology to describe a distinct change of color appearance when alexandrite is switched from being illuminated by daylight $(6500 \mathrm{~K})$ to incandescent light $(2856 \mathrm{~K})$ [7]. The alexandrite shows the same color if a blackbody and a gray body have the same temperature, because the relative spectral power distributions of a blackbody and a gray body are the same at the same temperature.

The typical spectral transmittance of an alexandrite crystal has two bands in the visible spectrum [7]. Figure 1 illustrates the typical two-band spectrum of an alexandrite crystal. When the radiation of a radiating body passes through the crystal, the crystal appears different colors at different temperatures of the radiating body.

The continuous color sequence of a thermal radiator is displayed as the Planck locus in the CIE color diagram, from red, orange, yellow, and white to blue as temperature increases. In fact, the temperature of the thermal radiator only relates to the hue red to blue, regardless of it lightness and saturation, because the thermal radiator always has a lightness of 100 and a saturation of 0 as a light source regardless of its temperature.

The color of alexandrite at different temperature is represented by the hue-angle in the CIELAB color space, and can be calculated by

$$
\begin{aligned}
& X=\int \bar{x}(\lambda) s(\lambda) P(\lambda) d \lambda, \\
& Y=\int \bar{y}(\lambda) s(\lambda) P(\lambda) d \lambda, \\
& Z=\int \bar{z}(\lambda) s(\lambda) P(\lambda) d \lambda,
\end{aligned}
$$

where $X, Y$, and $Z$ are the tristimulus values, $\bar{x}(\lambda), \bar{y}(\lambda)$, and $\bar{z}(\lambda)$ are CIE color-matching functions; $s(\lambda)$ is the spectral power distribution of a radiating body; $P(\lambda)$ is the spectral

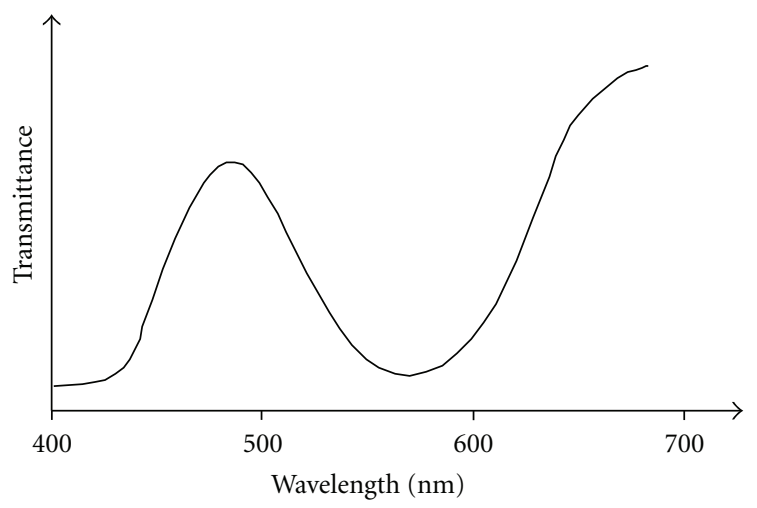

Figure 1: The typical spectral transmittance of alexandrite crystal in the visible wavelength range.

transmittance of the alexandrite; and $\lambda$ is the wavelength in the visible range. Then, the three coordinates of the CIELAB color space can be calculated as follows:

$$
\begin{aligned}
& L^{*}=116\left(\frac{Y}{Y_{n}}\right)^{1 / 3}-16, \\
& a^{*}=500\left[\left(\frac{X}{X_{n}}\right)^{1 / 3}-\left(\frac{Y}{Y_{n}}\right)^{1 / 3}\right], \\
& b^{*}=200\left[\left(\frac{Y}{Y_{n}}\right)^{1 / 3}-\left(\frac{Z}{Z_{n}}\right)^{1 / 3}\right],
\end{aligned}
$$

where $X_{n}, Y_{n}$, and $Z_{n}$ are the tristimulus values of the measured radiating body. Finally, the hue-angle $h$ in CIELAB space is given by

$$
h_{a b}=\tan ^{-1}\left(\frac{b^{*}}{a^{*}}\right) \text {. }
$$

Figure 2 illustrates the relationship between temperature of the thermal radiator and the hue-angle of the alexandrite crystal in the CIELAB color space. Temperature is a function of the hue-angle that can be determined by mathematical methods [8]. A polynomial function to the sixth power of hue-angle is usually adequate for most temperature measurement applications

$$
T=a_{0}+a_{1} h+a_{2} h^{2}+a_{3} h^{3}+a_{4} h^{4}+a_{5} h^{5}+a_{6} h^{6} .
$$

In a small temperature range, a polynomial function to the third power of hue-angle is sufficient.

The alexandrite effect spectropyrometer [8] consists of an optical probe, a spectrometer and a computer with a digital alexandrite effect filter, which tabulates the spectral transmittance along the a-crystallographic axis of the alexandrite with a maximum hue change of about 180 degrees in CIELAB color space between $6500 \mathrm{~K}$ and $2856 \mathrm{~K}$. The spectropyrometer measures the spectral power distribution of a thermal plasma torch through the alexandrite filter and calculates the hue-angle to determine its thermal temperature. 


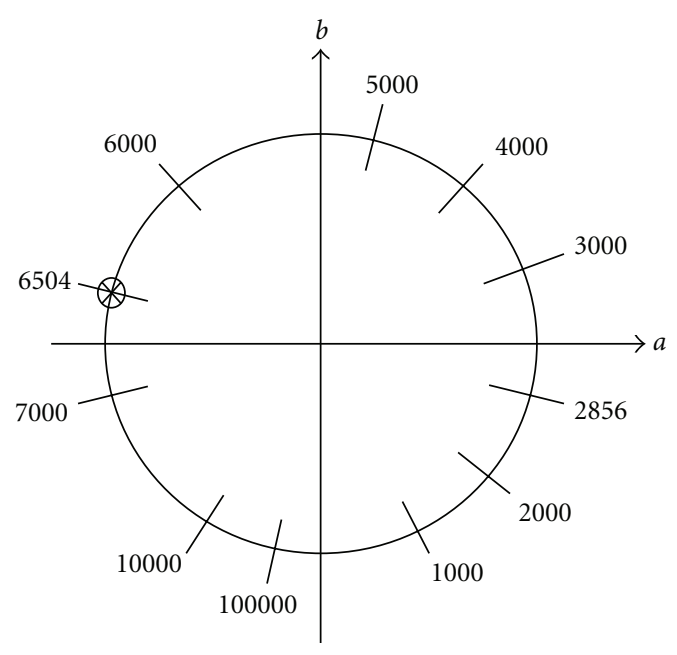

Figure 2: Relationship between the temperature and hue-angle in CIELAB color space.

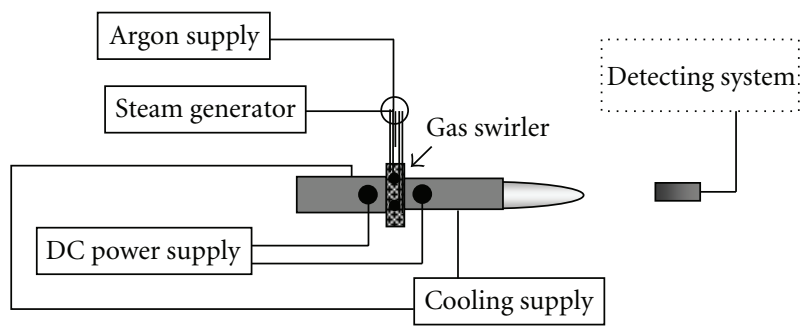

FIgURE 3: The layout of the nontransferred $100 \mathrm{~kW}$ dc plasma torch and temperature measurement setup.

\section{Experimental Setup}

The thermal temperature of a $100 \mathrm{~kW}$ DC plasma torch is measured (Figure 3). The plasma torch is operated with hollow electrodes. The gap between the two electrodes is $2 \mathrm{~mm}$ for easily initiating the electrical breakdown for working gas. The steel gas-swirler has four small annular holes of $2 \mathrm{~mm}$ diameter, which are bored with a neartangential slope towards the inside ring to make the injected gas form a swirl flow. The working gas is supplied through these holes from an argon or steam supply system into the electrodes gap in a vortex motion, which is necessary for arc stabilization. The rear electrode of torch is linked to negative polarity, and the working current I is adjusted from 100 to $200 \mathrm{~A}$, depending on the mass flow rate of argon or steam for a typical operating condition. In order to avoid influence by the cold particle scattering, the collimator was directed toward the nozzle of the plasma torch to measure the kinetic temperature of the plasma jet.

For comparison purposes, two temperature measurement methods are used: an alexandrite effect spectropyrometer with an extended temperature range of 1,800-50,000 K (LASP 4260, Liu Research Laboratories) and a conventional optical emission spectroscopy (OES) system. The wavelength range of the spectropyrometer is from 380 to $760 \mathrm{~nm}$ with a wavelength resolution of $1.0 \mathrm{~nm}$. The spectropyrometer can simultaneously provide a temperature and an emission spectrum of the plasma jet measured.

The OES system includes a collimator (74-UV, Ocean Opt.), a monochromator (Inspectrum 300, Princeton Instruments, wavelength resolution is $0.1 \mathrm{~nm}$ ), and a computer for acquiring the spectra of the plasma jet.

\section{Experimental Results}

Argon was used for the plasma torch, and the power is kept at $20 \mathrm{~kW}$ and current at $100 \mathrm{~A}$. The location of collimator, along the axis direction of the plasma, is $1.5 \mathrm{~m}$ away from the torch head. Figure 4 shows a measurement window of the spectropyrometer of the argon plasma. The temperature $(11,329 \mathrm{~K})$ and the emission spectrum are simultaneously displayed. The average measured thermal temperature is $11178 \pm 382 \mathrm{~K}$ for 12 measurements. The spectral power distribution consists of continuum underlying distribution and spectral lines.

In general, the state of high-pressure thermal plasma can be usually described as local thermodynamic equilibrium (LTE) or partial local thermodynamic equilibrium (PLTE). At the known transition probabilities, initial composition of thermal plasma, the temperature depends on the intensities of the spectral lines. The Ornstein method of measuring kinetic temperature on the basis of the ratio of the intensities of two spectral lines can be extended to a group of spectral lines belonging to the same type of atoms [10]. The Ornstein method can be expressed by the ratio of the intensities of the spectral lines

$$
\begin{aligned}
& \frac{\varepsilon_{i^{\prime}}}{\varepsilon_{i}}=\frac{(A g v)_{i^{\prime}}}{(A g v)_{i}} \exp \left(-\frac{E_{i^{\prime}}}{E_{i}}\right)=\frac{(A g v)_{i^{\prime}}}{(A g v)_{i}} \exp \left(-\frac{E_{i^{\prime}}-E_{i}}{k T}\right), \\
& \ln \left(\frac{\varepsilon}{A g v}\right)_{i}=y_{i}=C-\frac{E}{\mathrm{kT}},
\end{aligned}
$$

where $T$ is the kinetic temperature; $v$ is the emission frequency; $A$ is the transition probability; and $g$ is the statistical weight factor; $\varepsilon_{i}$ is the intensity of $i$ th spectral line; and $E_{i}$ is the energy associated with the $i$ th spectral line. To simplify the temperature calculation, we do not consider the absorption effect of the plasma. The temperature of plasma jet is estimated to be $13,410 \pm 500 \mathrm{~K}$ by the Ornstein method using the measured spectra of Ar I, as shown in Figure 5 with the parameters in Table 1.

The spectral power distribution of the plasma torch includes underlying continuum spectral power distribution and spectral lines. In fact, the underlying continuum spectral power distribution is attributed to the thermal emission of the plasma, and the spectral lines are caused by the electron transitions between specific energy levels of the atoms argon and copper, which is the nozzle material. If a plasma object is in the complete thermodynamic equilibrium, such as the Sun, its spectral power distribution is almost the same as that of blackbody at the same temperature. The directly measured thermal temperature in Figure 4 has an error caused by the present of spectral lines. 


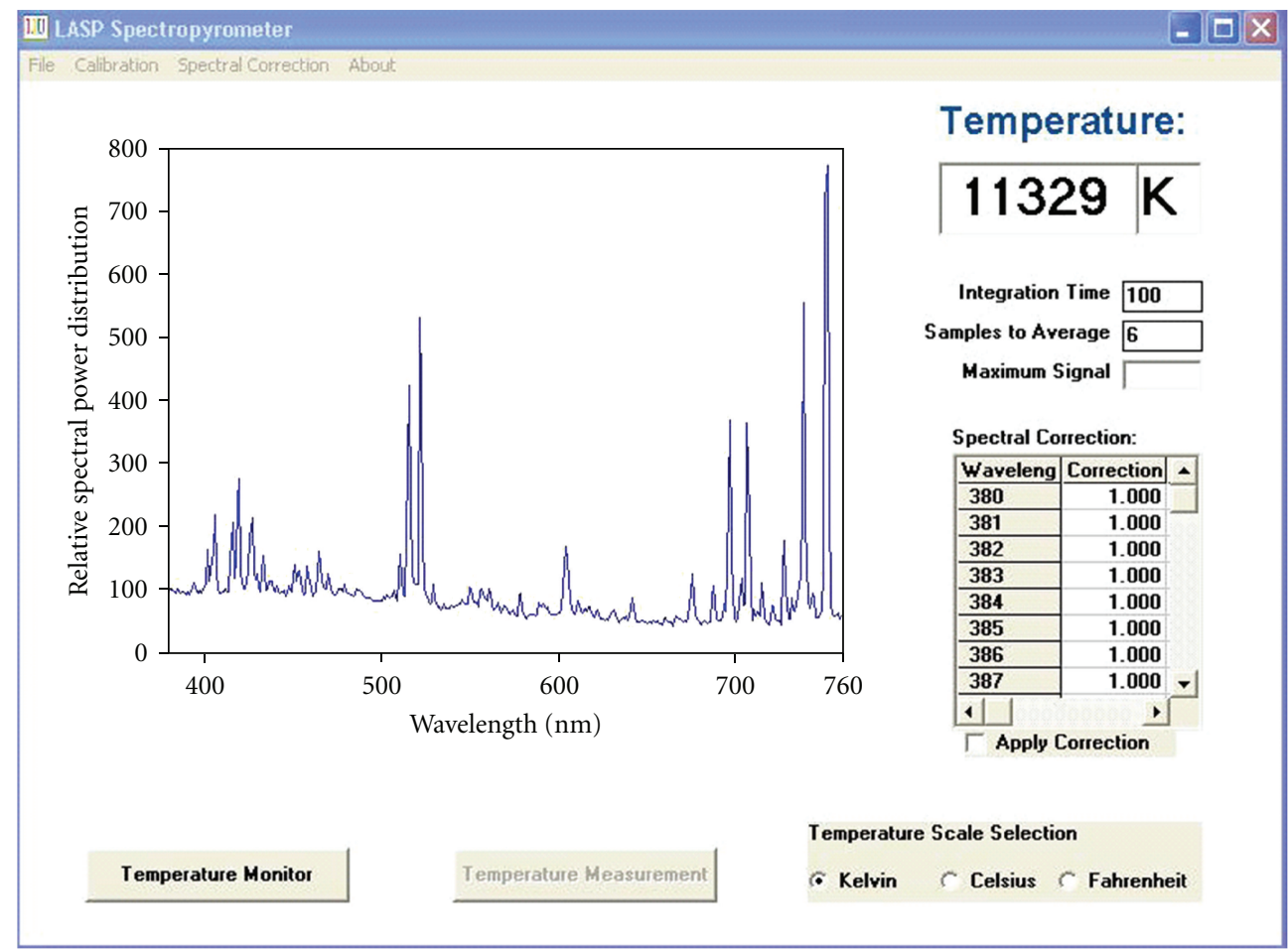

FIGURE 4: A temperature measurement of the plasma torch by the alexandrite effect spectropyrometer.

TABLE 1: The kinetic temperature of the argon plasma torch calculated by the Ornstein method.

\begin{tabular}{lcccccc}
\hline Wave-length & $\mathrm{A}(\mathrm{l} / \mathrm{s})$ & $g$ & $N($ counts $)$ & $E_{i}(\mathrm{ev})$ & $\mathrm{Ek}(\mathrm{ev})$ & 13.1531 \\
\hline 810.37 & $2.50 \mathrm{E}+07$ & 3 & 50989.5 & 11.6236 & 11.7232 & 13.2826 \\
794.82 & $1.86 \mathrm{E}+07$ & 1 & 32775.5 & 35793 & 11.8281 & 13.2826 \\
852.14 & $1.39 \mathrm{E}+07$ & 3 & 41350 & 11.6236 & 13.3022 & 10.097 \\
738.4 & $8.47 \mathrm{E}+06$ & 3 & 48011 & 11.8281 & 13.3279 \\
826.45 & $1.53 \mathrm{E}+07$ & 3 & 42051 & 11.8281 & 13.4799 & 9.3869 \\
750.39 & $4.45 \mathrm{E}+07$ & 3 & 19407 & 11.6236 & 14.5289 \\
426.63 & $3.12 \mathrm{E}+05$ & 3 & 15780 & 13.0757 & 15.4546 \\
521.05 & $1.10 \mathrm{E}+05$ & 7 & & & 9.5406 \\
\hline
\end{tabular}

Note A is transition probability, $g$ statistical weight factor, and $N$ counts of the spectral line.

The spectropyrometer has a spectral correction function, which can be used to correct the spectral power distribution by deleting the spectral lines in Figure 4 . The corrected spectral power distribution of the plasma is shown in Figure 6, and the thermal temperature is $9949 \mathrm{~K}$.

\section{Discussion}

The alexandrite effect spectropyrometer measures the spectral power distribution of a thermal radiator through the alexandrite filter, and calculates the hue-angle to determine the thermal temperature. The spectropyrometer can directly measure the thermal temperature of electric arcs and discharges, plasma jets, and high temperature combustion flames. The argon gas plasma temperature directly measured by the alexandrite effect spectropyrometer is $11178 \pm 382 \mathrm{~K}$, and the calculated kinetic temperature by the conventional Ornstein method is $13,410 \pm 500 \mathrm{~K}$. The temperature measured by the alexandrite spectropyrometer is lower (about $15 \%)$ than that calculated by the Ornstein method. The heat exchange efficiency between the plasma and gas particle (or ion) is overestimated for the plasma torch. Additionally, the process of photoionization, reabsorption and photoexcitation by excited particles of plasma cannot be ignored completely. Thus, the state of thermal plasma cannot reach the thermodynamic equilibrium. As stated by Fauchais et al. [4], the kinetic temperatures calculated from the spectra lines are generally overestimated due to the fact that the thermal plasma jet cannot be in true local thermodynamic equilibrium. These considerations exactly correspond with our measurement results. Considering the plasma jet is not in true thermal equilibrium and the directly measured thermal temperature by the alexandrite effect spectropyrometer is 


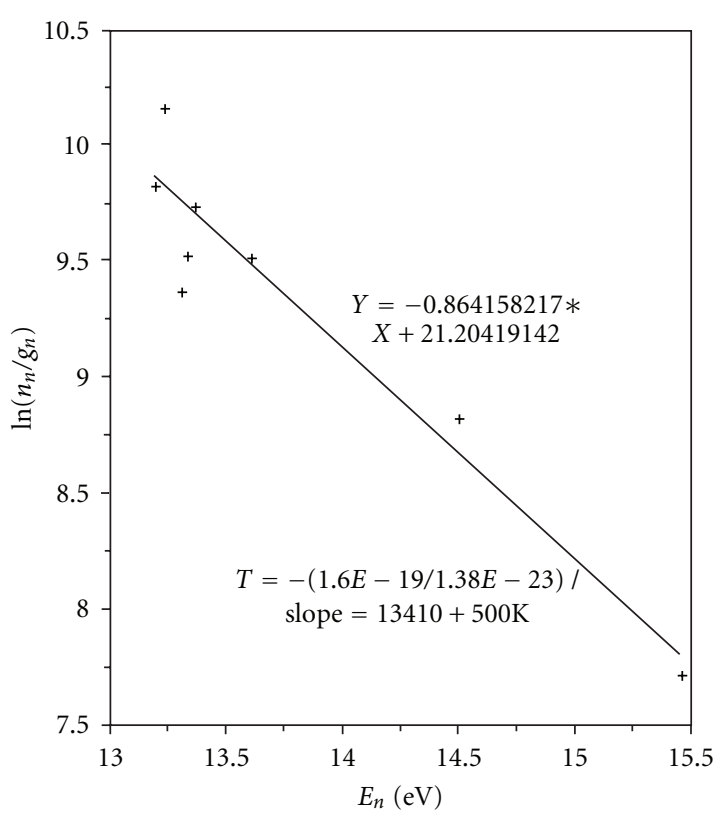

Figure 5: Temperature calculation of the plasma torch by the Ornstein method using the measured spectra of Ar I.

lower than that calculated by the OES method, the direct thermal temperature measurement by the alexandrite effect spectropyrometer is more precise than that measured by the OES method.

The very strong spectral lines of the plasma jet can cause an error for directly measuring thermal temperature by the spectropyrometer. However, the measurement error caused by the spectral lines can be corrected using the spectral correction function of the spectropyrometer. The thermal temperature calculated from the corrected continuum underlying spectral power distribution of the plasma jet is $9949 \mathrm{~K}$ with an estimated standard deviation of about $\pm 340 \mathrm{~K}$ (see Figure 6).

From Figure 6, we can know that the corrected spectral power distribution is approximately similar to that of blackbody in the visible wavelength range from 380 to $680 \mathrm{~nm}$. However, the spectral distribution slightly increases from 680 to $760 \mathrm{~nm}$, which is not normal for a thermal radiator. The increase of the spectral power distribution is caused by very strong electronic transitions of argon in this wavelength range, particularly at $696.5,706.7,738.4$, and $751.5 \mathrm{~nm}$. The very strong electronic transitions also contribute a small amount of continuum spectral power distribution in the wavelength range from 680 to $760 \mathrm{~nm}$.

An optically thick thermal plasma at high temperature can be approximately considered as a gray body, even as a blackbody, in the visible wavelength range $[11,12]$. The relative spectral power distribution of a gray body is the same as that of a blackbody at the same temperature. Figure 7 shows the further corrected spectral power distribution of the plasma jet according to that of the relative spectral power distribution of a blackbody. The calculated thermal temperature changes to $10106 \mathrm{~K} \pm 345 \mathrm{~K}$, which is slightly higher than the calculated thermal temperature of $9949 \mathrm{~K}$ $\pm 340 \mathrm{~K}$ in Figure 6 . The thermal temperature calculated from the further corrected spectral power distribution in Figure 7 is the true thermal temperature of the measured plasma jet, and it is more accurate than that calculated from the corrected spectral power distribution in Figure 6. However, the difference between the two corrected thermal temperatures is about $1.5 \%$, which is not significant for the thermal temperature measurement of the plasma jet.

Although the corrected spectral distributions are still in a zigzag shape, the zigzag-shaped spectral power distribution has little effect on the accuracy of calculating the thermal temperature due to the calculation of the tristimulus values of the spectropyrometer is an integration calculation in the whole measured wavelength range.

The true thermal temperature is lower than the directly measured thermal temperature, and the temperature different is about $12 \%$. Generally, the $12 \%$ difference is acceptable for on-line temperature measurement of a plasma jet in practical applications. However, to accurately measure the thermal temperature of a thermal plasma jet, it is very important to optimize the design and operation conditions of the thermal plasma torch. As shown in this paper, the alexandrite effect spectropyrometer is accurate and capable of monitoring the temperature variation of a plasma jet, which is a significant benefit to increase the reliability of a thermal plasma torch.

In fact, the stronger the spectral lines, the less accurate the directly measured thermal temperature by the spectropyrometer. The ratio between the directly measured thermal temperature and the corrected true thermal temperature can be used to indirectly estimate the thermal equilibrium state of the thermal plasma jet. The ratio of the true thermal temperature to the directly measured temperatures is a measure of how well the plasma approximates a blackbody, and the ratio is defined as the blackbody level (BL) of a thermal plasma

$$
\mathrm{BL}=\frac{\mathrm{TT}}{\mathrm{MT}}
$$

where TT is the calculated true thermal temperature (in Kelvin) and MT is the directly measured thermal temperature (in Kelvin). When the BL of a thermal plasma is 1, such as that of the Sun, the thermal plasma is a blackbody in the state of complete thermodynamic equilibrium. When the $\mathrm{BL}$ of a thermal radiator is less than 1 , it does not reach the complete thermal equilibrium. The smaller the BL is, the less the thermal plasma reaches the complete thermal equilibrium. The $\mathrm{BL}$ of the thermal plasma jet is

$$
\mathrm{BL}=\frac{10106 \mathrm{~K}}{11329 \mathrm{~K}}=0.892
$$

In general, the directly measured thermal temperature is always higher than the true thermal temperature calculated by the corrected spectral power distribution of a thermal plasma, thus the BL is always less than 1. 


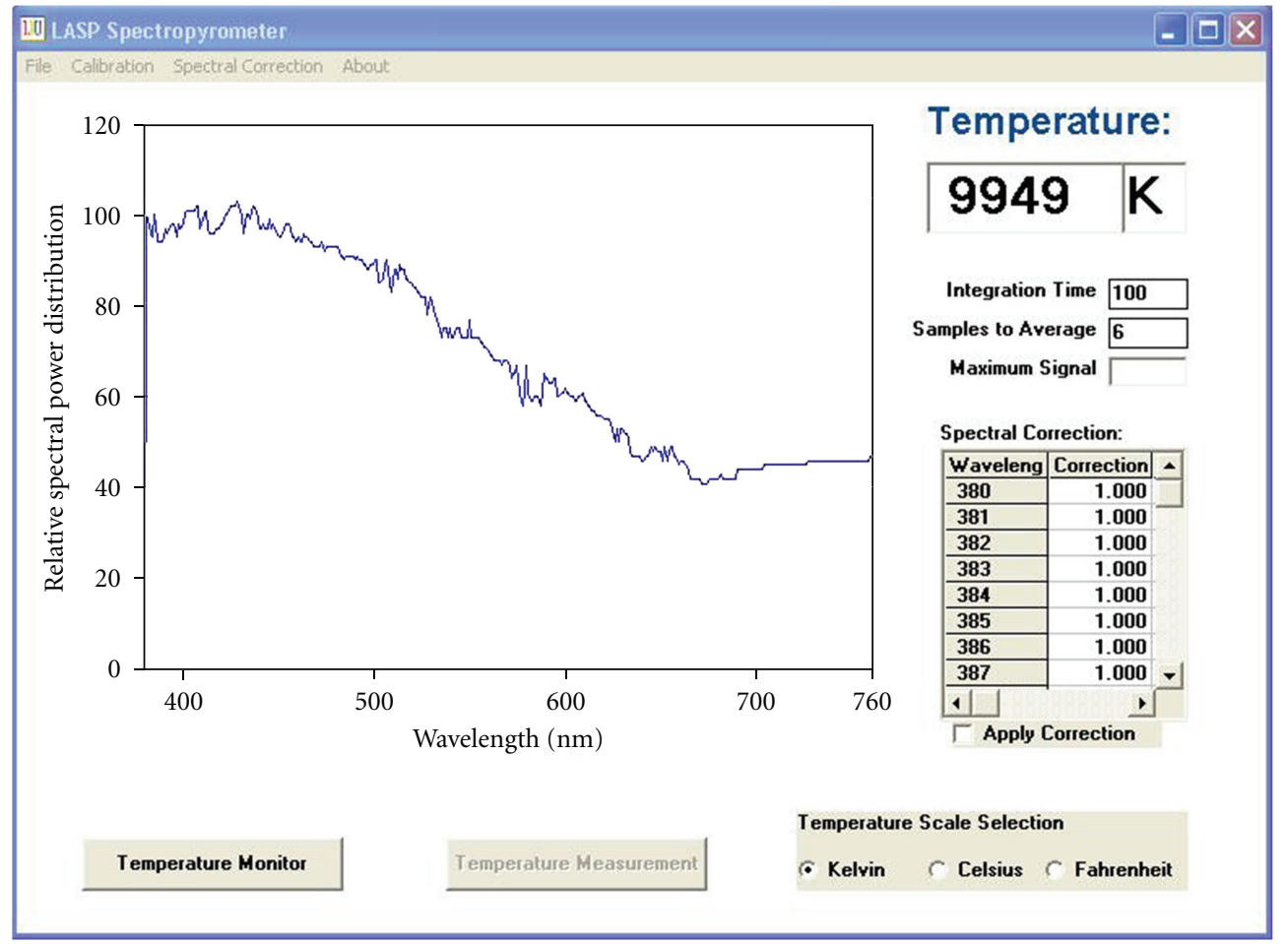

Figure 6: The corrected spectral power distribution of the plasma jet.

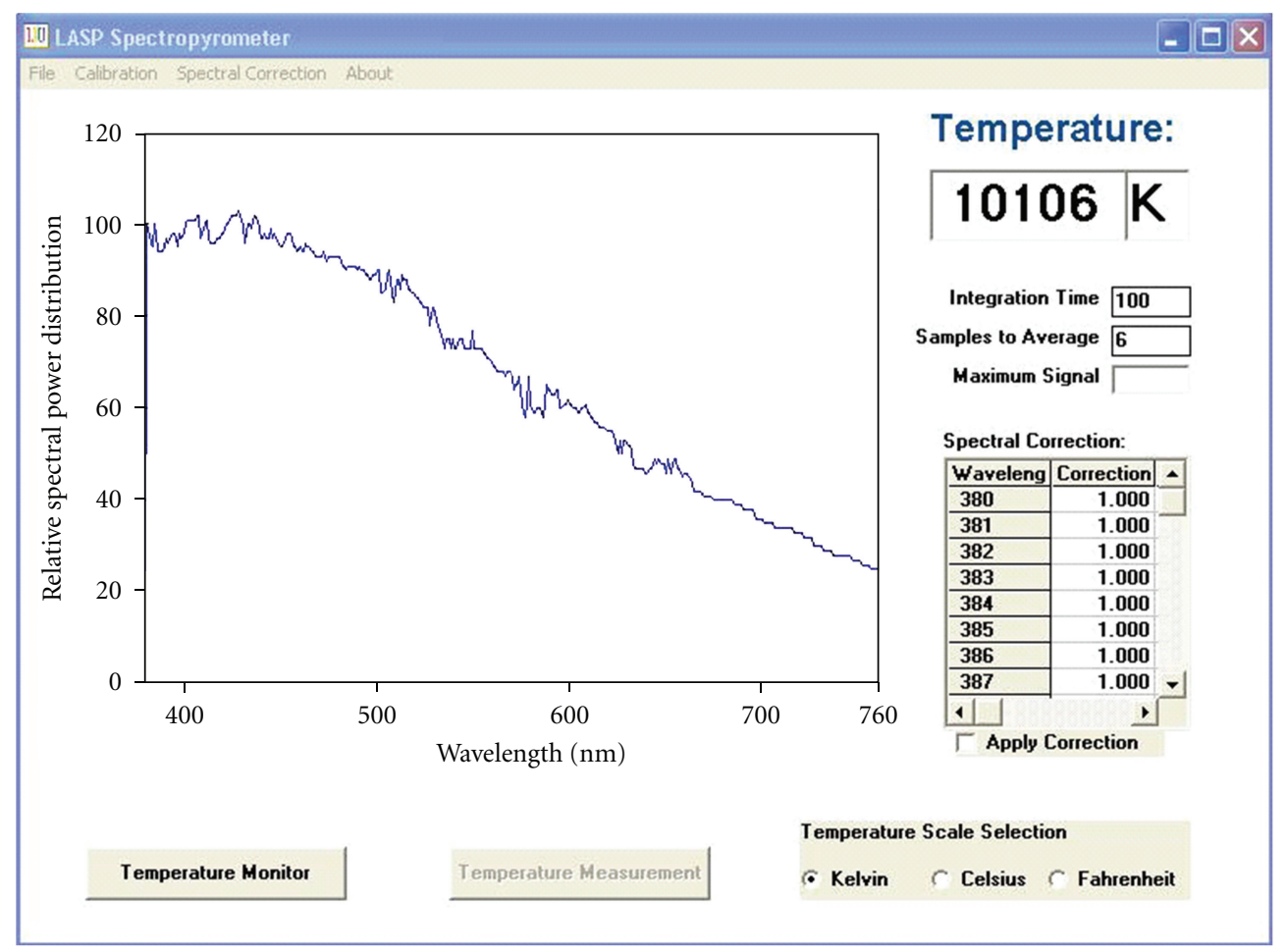

FIGURE 7: Corrected spectral power distribution according to that of thermal radiator. 


\section{Summary}

The alexandrite effect spectropyrometer is used to directly measure the thermal temperature of a $100 \mathrm{~kW}$ DC plasma torch, and the average measured thermal temperature is $11178 \pm 382 \mathrm{~K}$ for 12 measurements. The true thermal temperature is also calculated by deleting the spectral lines and correcting the underlying continuum spectrum of the measured spectral power distribution using the spectral correction function, and its true thermal temperature is $10106 \pm$ $345 \mathrm{~K}$. The difference between the directly measured thermal temperature and the true thermal temperature is about $1223 \mathrm{~K}$. In practical applications, the accuracy of directly measured thermal temperature by the spectropyrometer is accurate enough for scientific research and industrial applications. By using the spectral correction, the true thermal temperature can be obtained.

The directly measured thermal temperature of $11178 \pm$ $382 \mathrm{~K}$ is approximately consistent with the measured kinetic thermal temperature of $13,410 \pm 500 \mathrm{~K}$ by the OES method. In fact, the directly measured thermal temperature by the spectropyrometer is more precise than the kinetic temperature measured by the OES method to characterize the thermal property of the plasma torch. Furthermore, the true thermal temperature can be obtained by the spectropyrometer, but not by the OES method if the thermal equilibrium is not reached.

The accuracy of directly measured thermal temperature by the spectropyrometer depends on the state of thermal equilibrium. The weaker the spectral lines caused by electronic transitions are, the higher the accuracy of the thermal temperature measurement directly measured by the spectropyrometer. In other words, the closer to a blackbody radiator a measured plasma jet is, the higher the accuracy of the directly measured thermal temperature by the alexandrite effect spectropyrometer.

The blackbody level of the thermal plasma jet is defined as the ratio of the true thermal temperature to the directly measured thermal temperature by the spectropyrometer. The higher the blackbody level, the more the thermal plasma jet approaches a blackbody. The BL can be used to indirectly estimate the thermal equilibrium level of the thermal plasma jet or any other types of thermal plasma, since when a plasma reaches a complete thermal equilibrium, it will approach a blackbody.

\section{Acknowledgment}

The authors are indebted to the anonymous reviewer for providing helpful comments.

\section{References}

[1] C. Corliss, "Temperature of a copper arc," Journal of Reserch of the National Bureau of Standatds-A. Physics and Chemistry, vol. 66A, pp. 5-12, 1962.

[2] P. G. Slade and E. Schulz-Gulde, "Spectroscopic analysis of high-current free-burning ac arcs between copper contacts in argon and air," Journal of Applied Physics, vol. 44, no. 1, pp. 157-162, 1973.
[3] J. F. Key, J. W. Chan, and M. E. Mcllwain, "Process variable influence on arc temperature distribution," Welding Journal, vol. 62, no. 7, pp. 179-184, 1983.

[4] P. Fauchais, J. F. Coudert, M. Vardelle, A. Vardelle, and A. Denoirjean, "Diagnostics of thermal spraying plasma jets," Journal of Thermal Spray Technology, vol. 1, no. 2, pp. 117-128, 1992.

[5] M. Ballico, Temperature and Humidity Measurement, edited by R. Bentley, Springer, Singapore, 1998.

[6] K. Nassau, The Physics and Chemistry of Color, John Wiley \& Sons, New York, NY, USA, 2nd edition, 2001.

[7] Y. Liu, J. Shigley, E. Fritsch, and S. Hemphill, "'Alexandrite effect' in gemstones," Color Research \& Application, vol. 19, no. 3, pp. 186-191, 1994.

[8] Y. Liu, "Alexandrite effect spectropyrometer," in Photonic Devices and Algorithms for Computing VIII, vol. 6310 of Proceedings of SPIE, pp. 1-8, San Diego, Calif, USA, 2006.

[9] CIE, Publication No. 15.2, Colorimetry, (CIE Central Bureau, Vienna), 1996.

[10] A. Ovsyannikov and M. Zhukov, Plasma Diagnostics, Cambridge International Science Publishing, London, UK, 2000.

[11] M. Boulos, P. Fauchais, and E. Pfender, Thermal Plasmas, Plenum Press, New York, NY, USA, 1990.

[12] R. Siegel and J. Howell, Thermal Radiation Heat Transfer, McGraw-Hill, New York, NY, USA, 1981. 

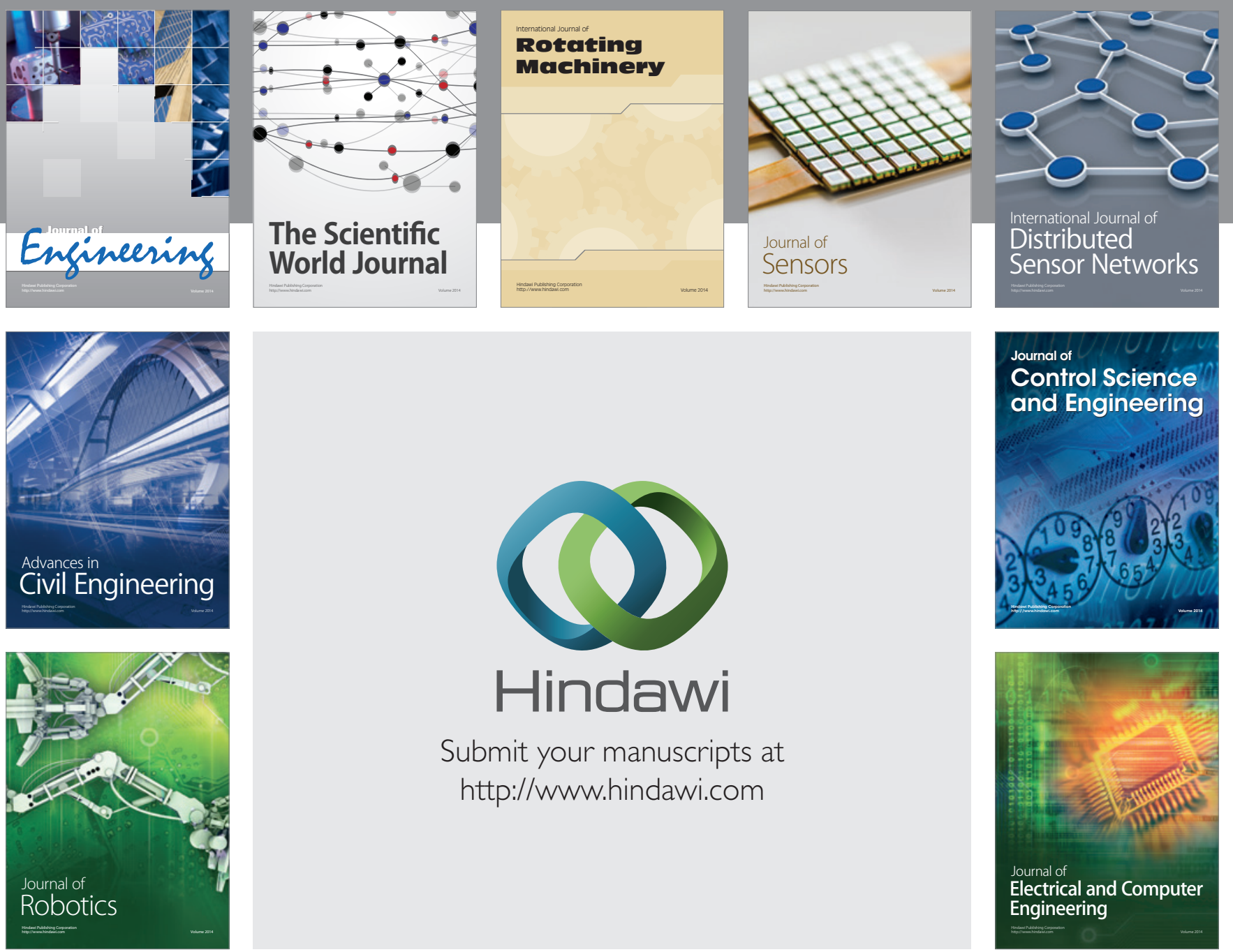

Submit your manuscripts at

http://www.hindawi.com
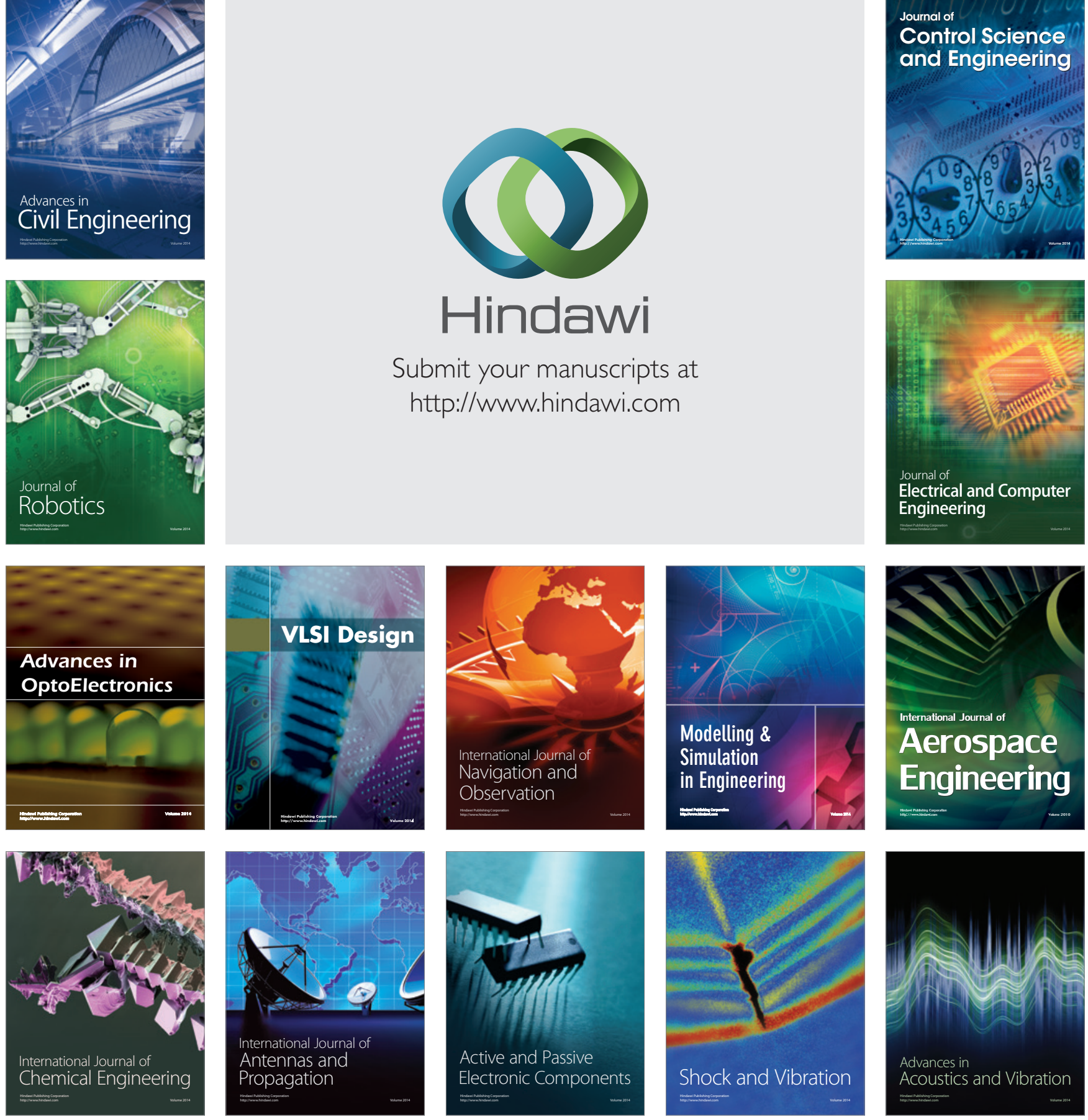